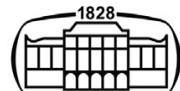

AKADÉMIAI KIADÓ

UNIVERSITY of DEBRECEN

\section{International Review of Applied Sciences and Engineering}

$13(2022) 1,22-28$

DOI:

$10.1556 / 1848.2021 .00263$

(c) 2021 The Author(s)

\title{
Numerical experimental comparison of mudflow by smoothed particle hydrodynamics (SPH)
}

\author{
Mahsa Bokharaeian ${ }^{1 *} \odot$, Reza Naderi ${ }^{2}$ and Árpád Csámer ${ }^{3}$ \\ ${ }^{1}$ Debrecen University, Hungary \\ ${ }^{2}$ Shahrood University of Technology, Iran \\ ${ }^{3}$ Debrecen University, Hungary
}

Received: March 9, 2021 • Accepted: July 1, 2021

Published online: August 27, 2021

\begin{abstract}
Flow-like landslides are a serious geologic hazard that can cause life and property loss all over the world. Mudflow is a kind of debris flow that has been classified as a non-Newtonian flow. The Smoothed particle hydrodynamics method (SPH) is a powerful tool for modeling fluids, such as debris/mudflows, which can be described in terms of local interactions of their constituent parts. In this paper, the Herschel-Buckley rheology model and SPH are used to simulate free-surface mudflow under the gate. The run-out distance and velocity of mudflow during the time are calculated with numerical simulation and compared with the laboratory result. Our results indicate the rate of increase of run-out and viscosity in the computer model is more than the experimental model and it is because of friction that is assumed to be zero. In the computer simulation, friction is exactly zero but in the experimental model, it could be measured and assumed zero. Finally, Abacus had a good result and can be used for mudflow simulation and protection of run-out distance and viscosity.
\end{abstract}

\section{KEYWORDS}

mudflow, Herschel-Buckley, smooth particle hydrodynamics, non-Newtonian flow

\section{INTRODUCTION}

In the last few decades, flow-like landslides (mudflow and debris flow) have been studied specialized, because these phenomena are very catastrophic and have resulted in so many victims and serious financial losses all over the world [1]. Approximately 300 million people all over the world are exposed to landslides with the greatest economic losses [2]. A supperlarge landslide occurred in the southeast of Tibet in April 2000 and formed a landslide dam over the Yigong River. Two months later, due to the failure of the landslide dam, a catastrophic flood occurred and caused a serious loss [3]. On 6 August 2010 in southwest British Columbia, Canada (Mount Meager), a large rock avalanche occurred. The landslide started as a rockslide and then the peak of Mount Meager collapsed. It was one of the largest landslides since 1998 in the territory of Capricorn Creek watershed and in the Mount Meager since 1930 but in this event, no lives were lost [4]. In 2013 a large-scale landslide occurred at Wulipo Sanxi village. It killed 44 and buried 117 people, as well as damaged many rural houses [5]. Another catastrophic landslide occurred in May 2014 in Afghanistan. It buried 86 houses and took almost 2,700 people's lives [6]. Nowadays, there are so many cases of landslides with great destructive effects, so the understanding and monitoring of landslide are necessary.

The term landslide includes a broad range of processes related to the movement of E-mail: mahsa.bokharaeian@science. unideb.hu various materials, such as rocks, soils, artificial fill, or their mixtures, down a slope under the direct influence of the gravitation. The materials can move in different ways such as sliding, falling, spreading, toppling, or flowing. Landslides can be classified based on the type of materials and the mechanism of movement. One of the most used classifications is described 
by Varnes [7, 1]. According to this classification, there are five types of movements: Fall (when rocks break and fall freely), Topple (created by rotating of units around a specific point), Slides (moving of the cohesive blocks along a defined surface of sliding), Spread (lateral extension caused by shear or tensile fractures) and Flow (movement of loose Earth material as a fluid) [7].

In this project, a special form of the landslide (mudflow) is considered. Mudflow can be regarded as a hyper-concentrated mixture of water and fine sediments that can be characterized by high concentrations of silt and clay. There are various reasons which initiate these flows, such as heavy rainfall, snowmelt, submarine landslides, dam break on sloping beds, human activities, and climate change [8]. Mudflow can destroy a range of structures like towns, factories, mines and cause serious damages to river protection structures and other hydraulic equipment as well as transporting large quantities of silt to the river cause decreasing water quality or even blocking rivers. In addition, mudflow can have a destructive effect on crops and features of the natural environment [9]. So, numerical simulation of mudflow can play an essential role to illuminate the mudflow's impact on the design and operation of hydraulic devices and to reduce its destructive effects.

\section{MATERIALS AND METHODS}

For a simulation of mudflow, it is necessary to know fluid behavior. Two main mathematical approaches are used to describe the behavior of multi-phase free surface flows such as mudflow. In the first method, which is more common, two separate simulations are used for the solid (sediment) and liquid (water) phases. In this method, the fluid phase governing equations in the model are continuity and momentum equations, while for sediment transport simulation an advection-diffusion model is used. However, this method suffers from some difficulties, such as the considerable computational effort required to solve the coupled equations. In the second method, the water/sediment mixture is treated as a non-Newtonian fluid [10].

In the case of the non-Newtonian fluid, shear stress and the rate of shear strain have a nonlinear relation. Various relationships and models have been proposed for these types of fluids, which are given in Table 1.

Table 1. Mathematical models for simulating the behavior of nonNewtonian fluids

\begin{tabular}{lc}
\hline equation & Rheology model \\
\hline$\tau=\tau_{0}+\mu_{p} \gamma$ & Bingham \\
$\tau=\tau_{0}+k \gamma^{n}$ & Herschel-Buckley \\
$\sqrt{\tau}=\sqrt{\tau_{0}}+\sqrt{\mu_{p} \gamma}$ & Casson \\
$\tau=k_{p l} \gamma^{n_{p l}}$ & Power Law \\
$\tau=k\left(\gamma_{0}+\gamma\right)^{n}$ & Robertson-Stiff \\
\hline
\end{tabular}

Where $\tau$ is the shear stress, $k$ is the consistency index, $\tau_{0}$ is the yield shear stress, $\gamma_{0}$ is a correction factor for the shear rate $\gamma, \mu_{p}$ is the plastic viscosity and $n$ is the flow index.

In this research, the Herschel-Buckley rheology model was used [11].

\subsection{Herschel-Buckley fluid}

Experimental results show that the Herschel-Bulkley model can be more appropriate in depicting the nonlinear properties of the stress tensor in mudflow and rheological data in a wide range of shear rates [12]. This model is a combination of the Bingham models and power-law and includes a yield stress value. The stress for describing the nonlinear viscoelastic behavior of mudflow is defined as:

$$
\tau= \begin{cases}0 & \tau<\tau_{\mathbf{B}} \\ \tau_{B}+\mu_{B}(\dot{\gamma})^{N} & \tau \geq \tau_{B}\end{cases}
$$

Where $\tau_{B}$ is the yield stress, $\mu_{B}$ is a consistency index and $N$ is the flow behavior index, respectively. $N$ and $\mu_{B}$ are usually determined based on experimental viscometer results. The shear strain rate in 2-D is calculated as:

$$
\dot{\gamma}=\left(2 S_{i j} S_{i j}\right)^{\frac{1}{2}}=\sqrt{2\left(\frac{\partial u}{\partial x}\right)^{2}+2\left(\frac{\partial v}{\partial y}\right)^{2}+\left(\frac{\partial u}{\partial y}+\frac{\partial v}{\partial x}\right)^{2}}
$$

$[8,13]$.

\subsection{Numerical simulation}

Numerical simulation is one of the most rapidly growing multidisciplinary fields in computer studies. This approach is regularly used to modeling and solving complex engineering and scientific problems. It is also a very useful tool to test various theories just before the laboratory and field investigations and may provide additional support in the interpretation and even the recognition of new phenomena. For example, powerful grid-based methods like finite difference methods (FDM) and finite element methods (FEM) play an important role in solving engineering problems in computational fluid dynamics (CFD) and computational solid mechanics (CSM) [14].

While each method has its advantages and disadvantages, it is very hard to establish ranks or superiority between them. For example, the finite difference method (FDM) is very effective in numerical solution procedures and it has advantages due to its shorter computing time and storage, but it needs a special boundary presentation and the results may not be accurate. Although for problems with any indiscriminate geometric shapes, FEM can be helpful, it needs more time for computing and storage compared to the FDM [15]. On the other hand, the effects and consequences of large deformation and post-failure events are still imperative research topics in the geomechanics area and are under debate. Numerical predictions of these phenomena can be useful and provide important information for engineering practice and design. However, neither the FEM nor the FDM seem to be suitable to provide large-scale and high-resolution flow analysis of 
geotechnical problems. FEM is a grid-based method and suffers from grid distortions caused in exactitude through the solution or even failure of the calculation of numerical integration due to negative values of Jacobian determinants at nodes. On the other hand, the DEM simulation does not have this limitation, but it is computationally expensive, and the processor power restricts the method to solve only small-scale simulations with a few hundred thousand particles, thus it is unable to handle large-scale problems. Besides, DEM implements a non-continuum approach, which means the equation of fluid constitutive cannot be applied [16, 17].

The mesh-free method (SPH) has a definite superiority in solving problems with large deformation and post-failure behavior compared to grid-based ones, such as FEM and DEM.

\subsection{Mesh-free methods}

Nowadays, due to the advantages of the mesh-free method in fluid flow simulating with a free surface, more attention has been paid to this method [18]. However, the mesh-free method does not use any mesh like the grid-based methods, and it is just based on a set of randomly distributed nodes. Mesh-free methods can analyze the movement of boundaries and connections much easier than the FEM because it needs a lot of time to fit and move a mesh continuously which is not effective. Besides, for mesh-free methods, the convection term derived from the movement of particles and numerical diffusion does not mean a problem instead of FDM [19].

Smoothed particle hydrodynamics (SPH) can be regarded as a particle-based method (mesh-free) that is used for continuum scale applications. The SPH method was first developed and applied in 1977 to solve three-dimensional astrophysical simulations. In this approach, the particles can move similarly to those in fluid flows (liquid or gas), so it can efficiently handle problems in the dynamic fluid. In the SPH, Lagrangian formalization is followed to compute the equations of fluid dynamics and position and physical variables of particles (e.g., velocity, density, pressure) are approximated by the values of the neighbour particles with a kernel function $[19,20]$.

SPH formulation is based on two fundamental steps, enabling it to cope with the free surface flow and large deformation problems without using grids.

\section{1. kernel approximation}

It can be estimated by average summation over the values of the nearest surrounding particles.

$$
f(x)=\int_{D} f\left(x^{\prime}\right) \delta\left(x-x^{\prime}\right) d x^{\prime}
$$

Where $f(x)$ is a function to define position of vector $x$ in the volume of integral $D$ that contains $x$, and $\delta\left(x-x^{\prime}\right)$ is the Dirac delta function given by:

$$
\delta\left(x-x^{\prime}\right)= \begin{cases}1 & x=x^{\prime} \\ 0 & x \neq x^{\prime}\end{cases}
$$

Since the Dirac delta function is used in equation (3), the integral representation is exact. If smoothing function
$W\left(x-x^{\prime}, h\right)$ is used instead of Delta function kernel $\delta\left(x-x^{\prime}\right)$, the function of $f(x)$ is given by:

$$
f(x)=\int_{\Omega} f\left(x^{\prime}\right) W\left(x-x^{\prime}, h\right) d x^{\prime}
$$

Where $W$ is called smoothing kernel function and $h$ is smoothing length that defines the influence area of the smoothing function. The smoothing kernel function has three conditions. The first one is the normalization condition:

$$
\int_{\Omega} W\left(x-x^{\prime}, h\right) d x=1
$$

The second condition is when the smoothing length approaches zero (Delta function property):

$$
\lim _{h \rightarrow 0} W\left(x-x^{\prime}, h\right)=\delta\left(x-x^{\prime}\right)
$$

And the third condition is the compact condition:

$$
W\left(x-x^{\prime}\right)=0 \quad \text { for }\left|x-x^{\prime}\right|>K h
$$

Where $K$ is a constant that defines the effective area of the smoothing function [14].

2. particle approximation

The value of a function at a special particle $(i)$ can be approximated by using the average of those values of the function at all the particles in the support domain of particle $i$ weighted by the smoothing function.

$$
f\left(x_{i}\right)=\sum_{j=1}^{N} f\left(x_{j}\right) \frac{m_{j}}{\rho_{j}} W\left(x_{i}-x_{j}^{\prime}, h\right)
$$

Where $\rho(x)$ is the density of the particle, $m_{j}$ is the mass of particle $j, N$ is the number of particles within the support domain of particle $i, h$ is the smoothing length and $W$ is the kernel [21].

\subsection{Governing equations}

The mathematical model for non-linear materials and large deformation problems consists of:

a) continuity equation

$$
\frac{D \rho}{D t}+\rho \frac{\partial v^{\beta}}{\partial x^{\beta}}=0
$$

b) momentum equation

$$
\frac{D v^{\alpha}}{D t}-\frac{1}{\rho} \frac{\partial \sigma^{\alpha \beta}}{\partial x^{\beta}}-F=0
$$

c) energy equation

$$
\frac{D e}{D t}-\frac{\sigma^{\alpha \beta}}{\rho} \frac{\partial v^{\alpha}}{\partial x^{\beta}}=0
$$

Where $v$ is velocity, $\rho$ is the density, $\sigma$ is the stress tensor, which is related to the resolved strain rate tensor: 


$$
\sigma^{\alpha \beta}=-P \delta^{\alpha \beta}+\tau^{\alpha \beta}
$$

Where $P$ is the pressure, $\tau$ is the Shear stress and $\delta^{\alpha \beta}$ is the Dirac delta function:

$$
\delta\left(x-x^{\prime}\right)= \begin{cases}1 & x=x^{\prime} \\ 0 & x \neq x^{\prime}\end{cases}
$$

[1].

\subsection{Simulation}

To simulate the dam break and verification result, we used experimental results. In the experiment, a rectangular channel is used and with installing a camera in front of the channel some pictures from the flow are recorded as shown in Fig. 2 [22]. The same channel is simulated in Abaqussoftware (version 6.14). Dimensions of this channel are $4.8 \mathrm{~m}$ long, $0.3 \mathrm{~m}$ high, and $0.3 \mathrm{~m}$ wide as shown in Fig. 1 . A Plexiglas valve is used which is located at a distance of $0.5 \mathrm{~m}$ from the end of the channel. The thickness of this valve is about $0.02 \mathrm{~m}$, which is moving upward at a speed of $0.4 \mathrm{~m}$ per second. The height of the mud column behind the valve is $0.2 \mathrm{~m}$. In this simulation, bentonite-mud is used as a nonNewtonian and single homogeneous fluid, as detailed in Table 2. To simulate non-Newtonian flow, the HerschelBulkley model is used and since Abaqus does not have this model as a default, the codes for this model are written and synced with Abacus software.

Since the number of particles in the SPH method has a great impact on the accuracy of solving and reducing the computational cost, to obtain accurate results the number of these particles should be calculated. For this purpose, the simulation is repeated with an increasing number of the particles till further increase had little effect on the results. In the first step, the friction in simulation is ignored because in
Table 2. Rheological parameters of globentonite

\begin{tabular}{lcccr}
\hline $\begin{array}{l}\text { n Flow } \\
\text { index }\end{array}$ & $\begin{array}{c}\text { Consistency } \\
\text { index }\end{array}$ & $\begin{array}{c}\text { Density } \\
\left(\mathrm{kg} / \mathrm{m}^{3}\right)\end{array}$ & $\begin{array}{c}\text { Stress } \\
\text { yields }(\mathrm{Pa})\end{array}$ & Fluid type \\
\hline 0.33 & 3.1 & 1138.28 & 9.242 & Globentonite \\
\hline
\end{tabular}

the experiment a glassy channel is used as shown in Fig. 2, and the friction is low.

\section{RESULT}

Figure 3-a shows a longitudinal view of the laboratory test that is the result of analysis of images taken with the camera at the time of $0.22 \mathrm{~s}, 0.26 \mathrm{~s}$, and $0.34 \mathrm{~s}$ [22], and Fig. 3-b shows the runout distance of mudflow in the Abacus software at the same time.

For a better realization and comparison, the maximum horizontal distances of the wavefront during the time in both simulated and experimental tests are drawn, as shown in Fig. 4. The best curve that is passed to these points and their equation is obtained using the mathematical equations.

According to the software and laboratory results and comparing the two graphs it can be concluded that the results are very close to each other. Figure 5 shows the speed of flow over time. In this graph, the effect of friction on the flow is more visible. In the first part of the graph, the speed of flow is high, and the effect of friction is less, but over time in the computer simulation the speed is increasing at a constant rate, whereas in a laboratory test it is decreasing slowly.

To find out how much results can be affected by friction for the second step in the simulation, we used friction.

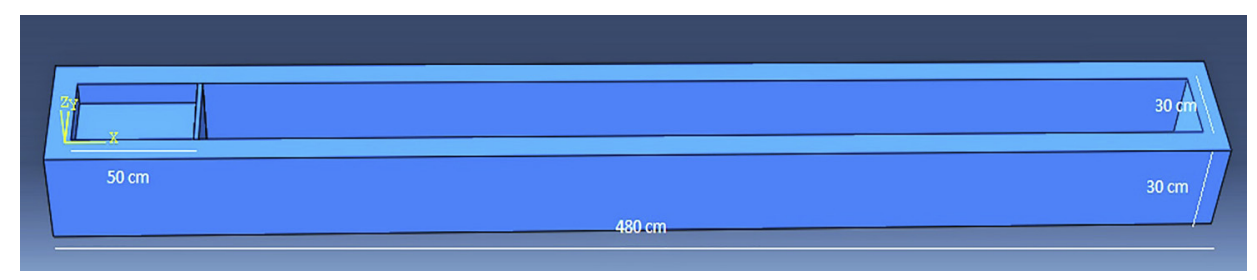

Fig. 1. Dimension of the simulated dam

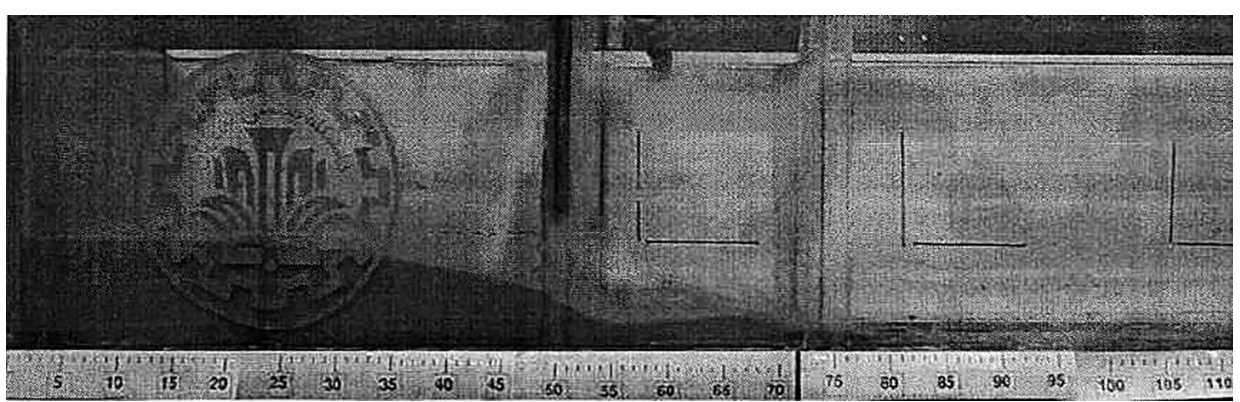

Fig. 2. Stream image of globentonite after opening the valve [22] 


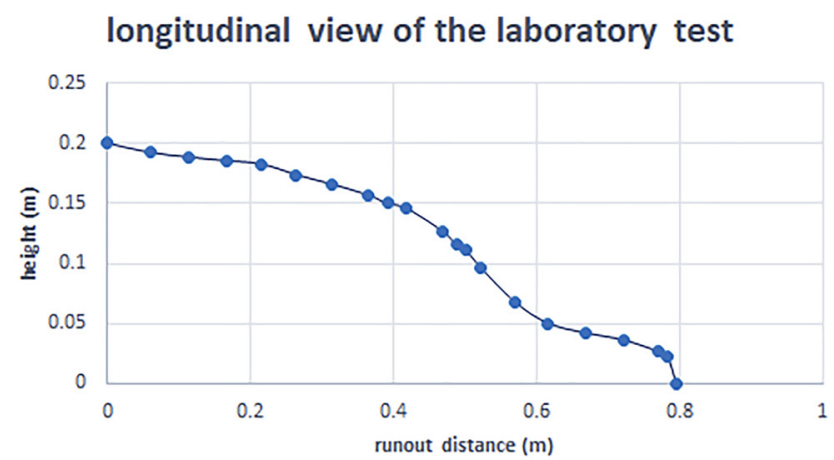

longitudinal view of the laboratory test

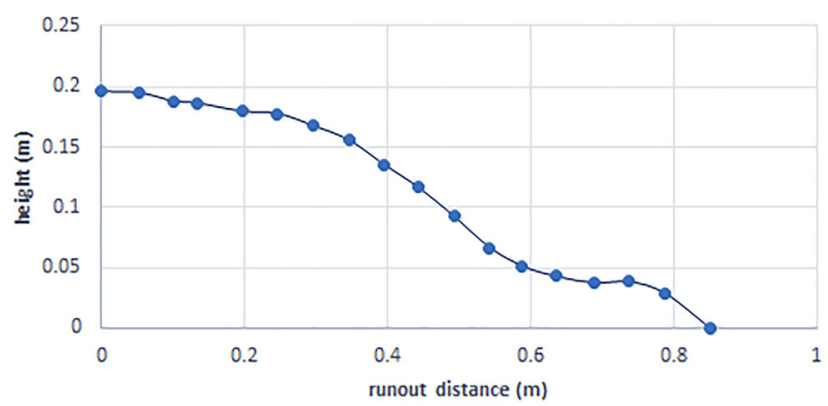

\section{longitudinal view of the laboratory test}

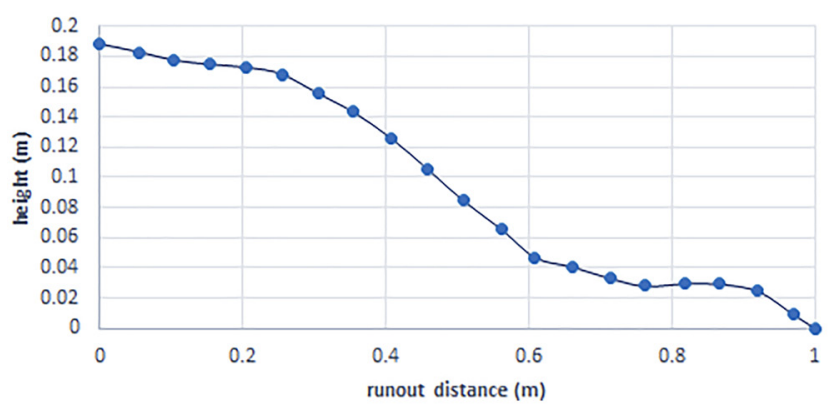

(a)

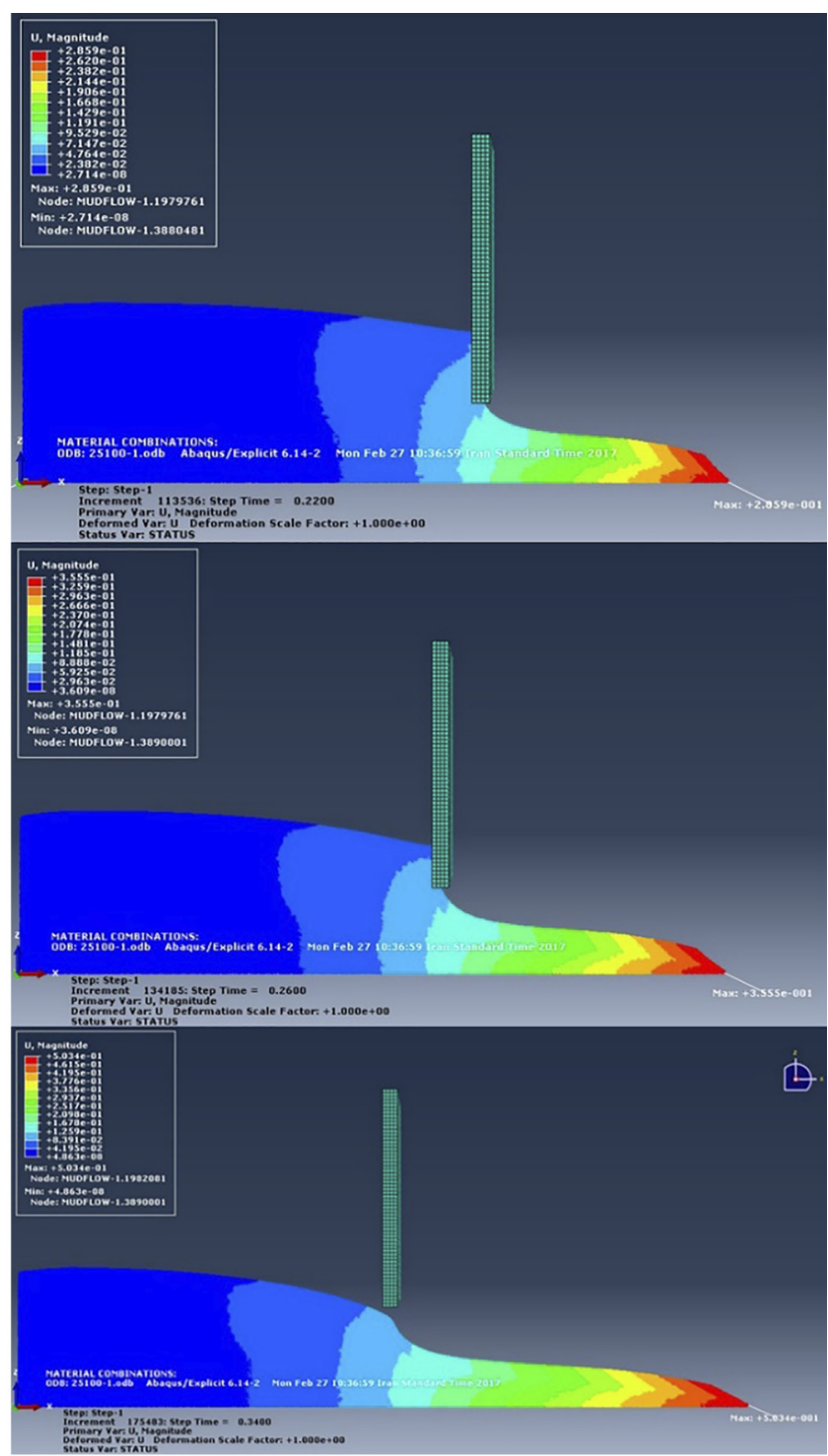

(b)

Fig. 3. Runout distance of mudflow at the time of $0.22 \mathrm{~s}, 0.26 \mathrm{~s}$ and $0.34 \mathrm{~s}$. a) laboratory test results, b) computer simulation results

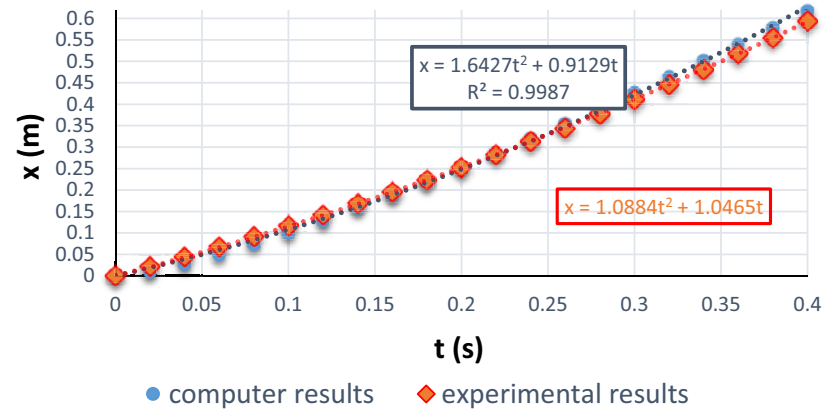

Fig. 4. Runout distances over time

Since in the experiment the real friction was not calculated, the coefficient of friction 0.01 is assumed. Figure 6 shows the maximum horizontal distances of the wavefront over time. In this case, computer results are more compatible

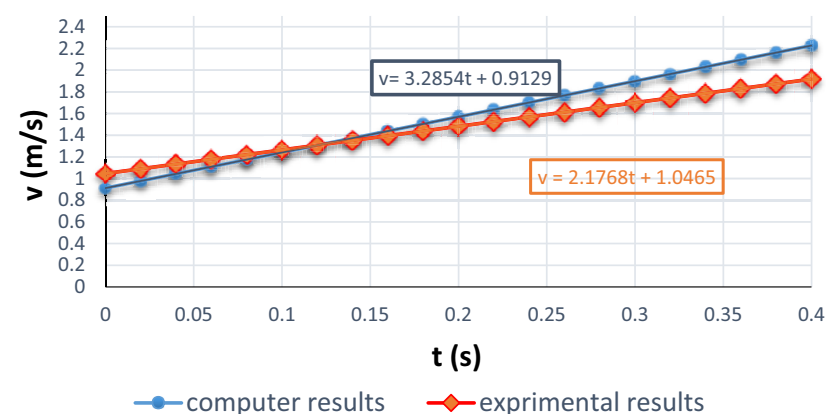

Fig. 5. Flow velocity over time

with laboratory results. Figure 7 shows the speed of flow over time, and it better shows the effect of friction. In the computer result like the experimental result, speed is decreasing slowly over time. In this case, the percentage of 


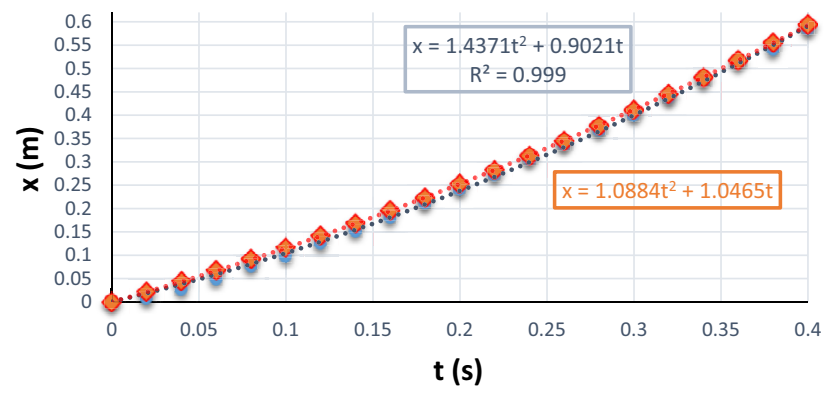

- computer results $\diamond$ experimental results

Fig. 6. Runout distances over time

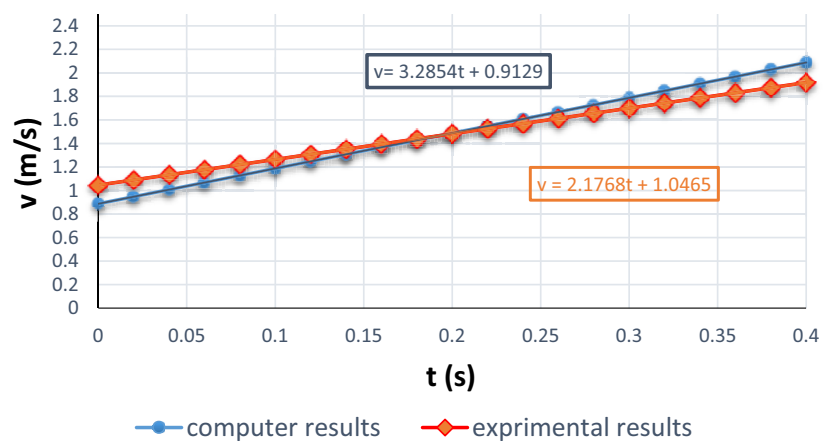

Fig. 7. Flow velocity over time

average error without using friction was $2.23 \%$ and with friction decreased to $1.15 \%$. So, in some cases, that speed is so high, friction has less effect on the flow but if we can measure the friction, the result will be more exact. On the other hand, in real mudflow speed is very high and friction has less effect so this simulation can be useful for predicting flow-affected areas.

\section{CONCLUSION}

The numerical technique is capable of handling complex flow cases such as submerged and regular hydraulic jumps after a gate. Further, the multi-phase problem was simulated properly in mudflow gravitational circulation caused by a submarine landslide. To compare with FVM and other traditional numerical methods, SPH is very convenient for simulating free surface mudflow in cases with large deformations. The laboratory and computational results are almost the same, and the reason for the small difference in results is due to friction avoidance. So, we can calculate that the SPH method and Herschel-Buckley model are powerful tools for the simulation of multi-phase problems, especially mudflow. Nowadays, with the increasing human activity and climate change, the possibility of mudflow occurrence has increased. So, it is necessary to find a solution to decrease damage caused by a mudflow. This achievement shows numerical simulation can be used to prevent the possibility of destruction and can be useful to prevent financial losses and to save human lives. In this project, we used Abaqus software that is a powerful tool to simulate different issues and can have a connection with other software, so it can be used to simulate mountains or slopes with a high possibility of mudflow. One of the most important abilities of this software is the ease of using the SPH method to simulate.

\section{REFERENCES}

[1] S. Cola, N. Calabrò, and M. Pastor, "Prediction of the flow-like movements of Tessina landslide by SPH model," in Landslides and Engineered Slopes. From the Past to the Future, Two Volumes+ CD-ROM, CRC Press, 2008, pp. 669-76.

[2] S. Almeida, E. A. Holcombe, F. Pianosi, and T. Wagener, "Dealing with deep uncertainties in landslide modelling for disaster risk reduction under climate change," Nat. Hazards Earth Syst. Sci., vol. 17, no. 2, pp. 225-41, 2017.

[3] Y. Shang, Z. Yang, L. Li, Q. Liao, and Y. Wang, "A super-large landslide in Tibet in 2000: background, occurrence, disaster, and origin," Geomorphology, vol. 54, nos 3-4, pp. 225-43, 2003.

[4] R. H. Guthrie, P. Friele, K. Allstadt, N. Roberts, S. G. Evans, K. B. Delaney, D. Roche, J. J. Clague, and M. Jakob, "The 6 August 2010 Mount Meager rock slide-debris flow, Coast Mountains, British Columbia: characteristics, dynamics, and implications for hazard and risk assessment," Nat. Hazards Earth Syst. Sci., vol. 12, no. 5, pp. 1277-94, 2012.

[5] L. I. Jingtao, C. H. Yuliang, W. A. Jun, W. A. Youming, L. I. Bin, W. A. Meng, and Y. A. Lei, "Remote sensing investigation and formation mechanism on wulipo landslide of July 10, 2013 in Sanxi village, Dujiangyan, Sichuan province," 工程地质学报, vol. 22, no. 6, pp. 1194-203, 2014.

[6] J. Zhang, D. R. Gurung, R. Liu, M. S. Murthy, and F. Su, "Abe Barek landslide and landslide susceptibility assessment in Badakhshan Province, Afghanistan," Landslides, vol. 12, no. 3, pp. 597-609, 2015.

[7] G. D. Bathrellos, and H. D. Skilodimou, "Landslides, a recurrent phenomenon,' landslide hazard assessment in mountainous areas of central Greece," Zeitschrift für Geomorphologie, vol. 25, pp. 95-114, 2021.

[8] Z. Ghadampour, B. N. Taleb, M. R. Hashemi, A. H. Nikseresht, and S. P. Neill, "Numerical simulation of free surface mudflow using incompressible SPH," Iranian J. Sci. Technol. Transaction B - Eng., vol. 37, no. C1, pp. 77-95, 2013.

[9] H. Xiao, Z. Luo, Q. Niu, and J. Chang, "The 2010 Zhouqu mudflow disaster: possible causes, human contributions, and lessons learned," Nat. Hazards, vol. 67, no. 2, pp. 611-25, 2013.

[10] A. Leonardi, F. K. Wittel, M. Mendoza, and H. J. Herrmann, "Coupled DEM-LBM method for the free- surface simulation of heterogeneous suspensions," Comput. Part. Mech., vol. 1, no. 1, pp. 3-13, 2014.

[11] V. C. Kelessidis, and R. Maglione, "Modeling rheological behavior of bentonite suspensions as Casson and Robertson-Stiff fluids using Newtonian and true shear rates in Couette viscometry," Powder Technol., vol. 168, no. 3, pp. 134-47, 2006.

[12] X. Huang, and M. H. Garcia, "A Herschel-Bulkley model for mud flow down a slope,” J. Fluid Mech., vol. 374, pp. 305-33, 1998. 
[13] S. S. Bahrainian, A. Nabati, and E. Haji Davalloo, "Improved rheological model of oil-based drilling fluid for South-western Iranian Oilfields," J. Pet. Sci. Technol., vol. 8, no. 3, pp. 53-71, 2018.

[14] G. R. Liu, and M. B. Liu, Smoothed Particle Hydrodynamics: A Meshfree Particle Method. World Scientific, 2003.

[15] M. Ikegawa, M. Kaiho, and C. Kato, "FEM/FDM composite scheme for viscous incompressible flow analysis," Computer Methods Appl. Mech. Eng., vol. 112, nos 1-4, pp. 149-63, 1994.

[16] H. H. Bui, R. Fukagawa, K. Sako, and S. Ohno, "Lagrangian meshfree particles method $(\mathrm{SPH})$ for large deformation and failure flows of geomaterial using elastic-plastic soil constitutive model," Int. J. Numer. Anal. Methods Geomechanics, vol. 32, no. 12, pp. 1537-70, 2008.

[17] D. Basu, K. Das, R. Janetzke, and S. Green, "Numerical simulations of non-Newtonian geophysical flows using smoothed particle hydrodynamics (SPH) method: a rheological analysis," InASME Int. Mech. Eng. Congress Exposition, vol. 54921, pp. 155-64, 2011.
[18] L. Fu, and Y. C. Jin, "A mesh-free method boundary condition technique in open channel flow simulation," J. Hydraulic Res., vol. 51, no. 2, pp. 174-85, 2013.

[19] M. A. Nabian, and L. Farhadi, "Multiphase mesh-free particle method for simulating granular flows and sediment transport," $J$. Hydraulic Eng., vol. 143, no. 4, p. 04016102, 2017.

[20] W. Wang, G. Chen, Z. Han, S. Zhou, H. Zhang, and P. Jing, "3D numerical simulation of debris-flow motion using SPH method incorporating non-Newtonian fluid behavior," Nat. Hazards, vol. 81, no. 3, pp. 1981-98, 2016.

[21] D. A. Fulk, and D. W. Quinn, "An analysis of 1-D smoothed particle hydrodynamics kernels,” J. Comput. Phys., vol. 126, no. 1, pp. 165-85, 1996.

[22] N. Navid, Experimental and Numerical Modeling of Flow ClayWater Mixture (Bentonite) with SPH Model. Master Thesis, Esfahan University, 2012. 